


\title{
Métodos de realce de anomalias magnéticas e sua aplicação no delineamento de feições geofísicas na região da volta grande do rio Xingu-PA
}

\author{
Alessandra de Barros e Silva Bongiolo, LPGA/UFPR, Francisco José Fonseca Ferreira, LPGA/UFPR.
}

Copyright 2008, SBGf - Sociedade Brasileira de Geofísica

Este texto foi preparado para a apresentação no IV Simpósio Brasileiro de Geofísica, Brasília 14 a 17 de novembro de 2010. Seu conteúdo foi revisado pelo Comitê Técnico do IV SimBGf, mas não necessariamente representa a opinião da SBGf ou de seus associados. É proibida a reprodução total ou parcial deste material para propósitos comerciais sem prévia autorização da SBGt.

\section{Abstract}

This work shows the applicability of various methods of enhancement of magnetic anomalies to assist in geological mapping of the Amazon Basin, Brazil. In addition, testing a new method, this is based on the tilt derivative of the total horizontal gradient. Depths statistics obtained by spectral analysis and Euler Deconvolution was used to supplement the qualitative interpretation, which resulted in the tectonic framework of the study area.

\section{Introdução}

Métodos potenciais (gravimetria e magnetometria) são largamente empregados no delineamento do arcabouço de bacias sedimentares, sobretudo a do Amazonas, cujo conhecimento é limitado pela escassez de acessos, afloramentos e perfurações, aliado à densa cobertura vegetal que oculta às feições de relevo e dificulta as interpretações geológicas. A área de estudo está localizada na porção central do Estado do Pará, limitada pelas seguintes coordenadas geodésicas: $2^{\circ} 30^{\prime}$ e $3^{\circ} 50^{\prime}$ latitude Sul e $50^{\circ} 57^{\prime}$ e $52^{\circ} 36^{\prime}$ de longitude Oeste (Fig.1). É atravessada de Sul para Norte pelo rio Xingu e localizase na denominada "volta grande do rio Xingu". Possui como principal referência hidrográfica ao Norte o rio Amazonas, a Oeste o rio Tapajós e a Leste o rio Tocantins. O maior município da região é Altamira.

Geologicamente (Fig.1), a área envolve rochas cristalinas do Cráton Amazônico e sedimentares e ígneas da Bacia do Amazonas. O embasamento é constituído por rochas metavulcânicas e metassedimentares deformadas e metamorfizadas nas fácies xisto-verde a anfibolito, bem como por terrenos gnáissico-migmatíticos e granulíticos, todos da Província Maroni-Itacaiúnas (Tassinari \& Macambira 1999, 2004) de idade Paleoproterozóica, com evolução principal ocorrida no intervalo de 2,2 a 1,95 Ga. No âmbito das rochas proterozóicas estão presentes a Sequência Três Palmeiras, composta por um conjunto de rochas metavulcano-sedimentares que sustentam uma serra de orientação WNW-ENE; metatonalito Rio Bajacá; tonalito Brasil Novo, representado por granitóides com deformação dúctil na forma de batólitos irregulares com direção regional NW-SE; granodiorito Belo Monte com granitóides leuco a mesocráticos; Complexo Bacajaí representado por uma faixa metamórfica de alto grau na forma de granitóides catazonais intrusivos, orogênicos, gnaissificados, isotrópicos e com textura ígnea preservada; granodiorito Babaquara presente na forma de um corpo granitóide com relevo acidentado e alongado segundo um trend regional NW-SE; Suíte Intrusiva Araparí composta por charnoquitos e charnoenderbitos que ocorrem na forma de batólitos e stocks orogênicos e Suíte Intrusiva São Jorge onde ocorrem batólitos e plútons graníticos (monzo e sienogranitos), geralmente de formas alongadas segundo 0 trend regional NW-SE (CPRM 2008).

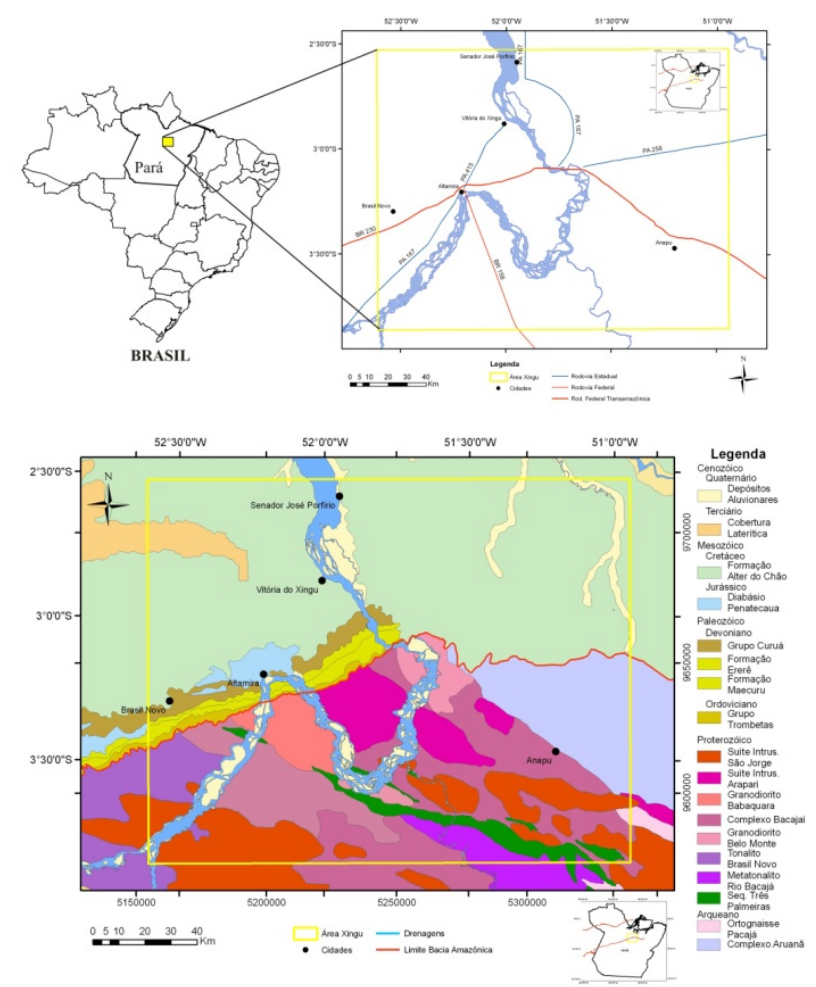

Figura 1 - Mapa de localização da área de estudo (painel superior) e mapa geológico (painel inferior).

Para Perico (2009) as rochas paleozóicas da Bacia do Amazonas que ocorrem na área de estudo correspondem ao Grupo Trombetas/Formação Manacapuru (arenitos intercalados a siltitos), à Formação Maecuru (arenitos finos a conglomeráticos, maciços e folhelhos subordinados), à Formação Ererê (siltitos maciços intercalados com folhelhos e arenitos). De idade mesozóica se destacam arenitos, siltitos e argilitos da Formação Alter do Chão e ainda diques e soleiras de diabásio (intrusões básicas). Coberturas lateríticas e 
cascalhos, areia, silte e argila de aluviões completam a coluna estratigráfica proposta por Cunha et al. (1994).

As principais estruturas que afetam a área são representadas por falhas NW-SE com variações para WNW-ESE, as quais possuem direções coincidentes às orientações das sequências vulcano-sedimentares do embasamento e também direções NE-SW, E-W, NNESSW e NNW-SSE representadas em rochas da bacia de maneira subordinada, de acordo com João et al. (1987) e com o Mapa Geológico do Estado Pará (CPRM 2008).

\section{Métodos/Problema Investigado}

A análise do arcabouço geofísico-estrutural da área foi apoiada no processamento e interpretação qualitativa e semiquantitativa de dados aeromagnetométricos disponíveis no LPGA/UFPR e organizados segundo malha regular de $1000 \times 1000$ metros e nivelados para uma altura de 1000 metros.

A definição das tendências geofísico-estruturais foi baseada em vários métodos de realce de anomalias de campos potenciais, como gradientes horizontais (Gx,Gy Cordell \& Grauch, 1985) e vertical (Gz - Evjen, 1936), gradiente horizontal total (Cordell \& Grauch, 1985), amplitude e inclinação do sinal analítico (Nabighian, 1972; Roest et al., 1992; Miller \& Singh, 1994), gradiente horizontal total da inclinação do sinal analítico (Verduzco et al., 2004), inclinação do sinal analítico do gradiente horizontal total (Ferreira et al., 2010, neste simpósio), dentre outros. Tais técnicas foram também aplicadas às malhas continuadas para cima $(2000,5000$ e 10000 metros), em correspondência ao incremento da ordem de derivação, no sentido de atenuar os ruídos e verificar a persistência das estruturas em profundidade.

Para estimativas de profundidades foram utilizados o espectro de potência radial (Spector \& Grant, 1970) e a Deconvolução de Euler (Thompson, 1982).

O problema investigado consiste em determinar, através dos métodos geofísicos propostos, contrastes e anomalias que possam associar-se a feições geológicas e estruturas do Cráton Amazônico e averiguar sua continuidade na Bacia do Amazonas.

\section{Resultados}

Os métodos de realce foram aplicados aos dados magnéticos continuados para 2000 metros, com o intuito de realçar as estruturas mais relevantes e eliminar possíveis ruídos. Os resultados obtidos podem ser observados na Figura 2.

A Figura 3 exibe o mapa magnético residual, no qual estão assinalados os principais domínios e lineamentos geofísicos. A definição dos domínios foi baseada nas intensidades das anomalias, enquanto os lineamentos foram obtidos através dos métodos de realce. Observase que o domínio A encerra as maiores amplitudes e se dispõe, juntamente com os lineamentos, segundo uma faixa de direção preferencial WNW-ESE, a qual penetra na bacia. O domínio B e seus lineamentos, por sua vez, mostram as menores intensidades segundo um corredor NE-SW situado na bacia. Os subdomínios A1 e B1 indicam, respectivamente, decréscimos e incrementos das intensidades dos domínios A e B. O domínio C, de amplitudes intermediárias, é disperso na área de estudo.
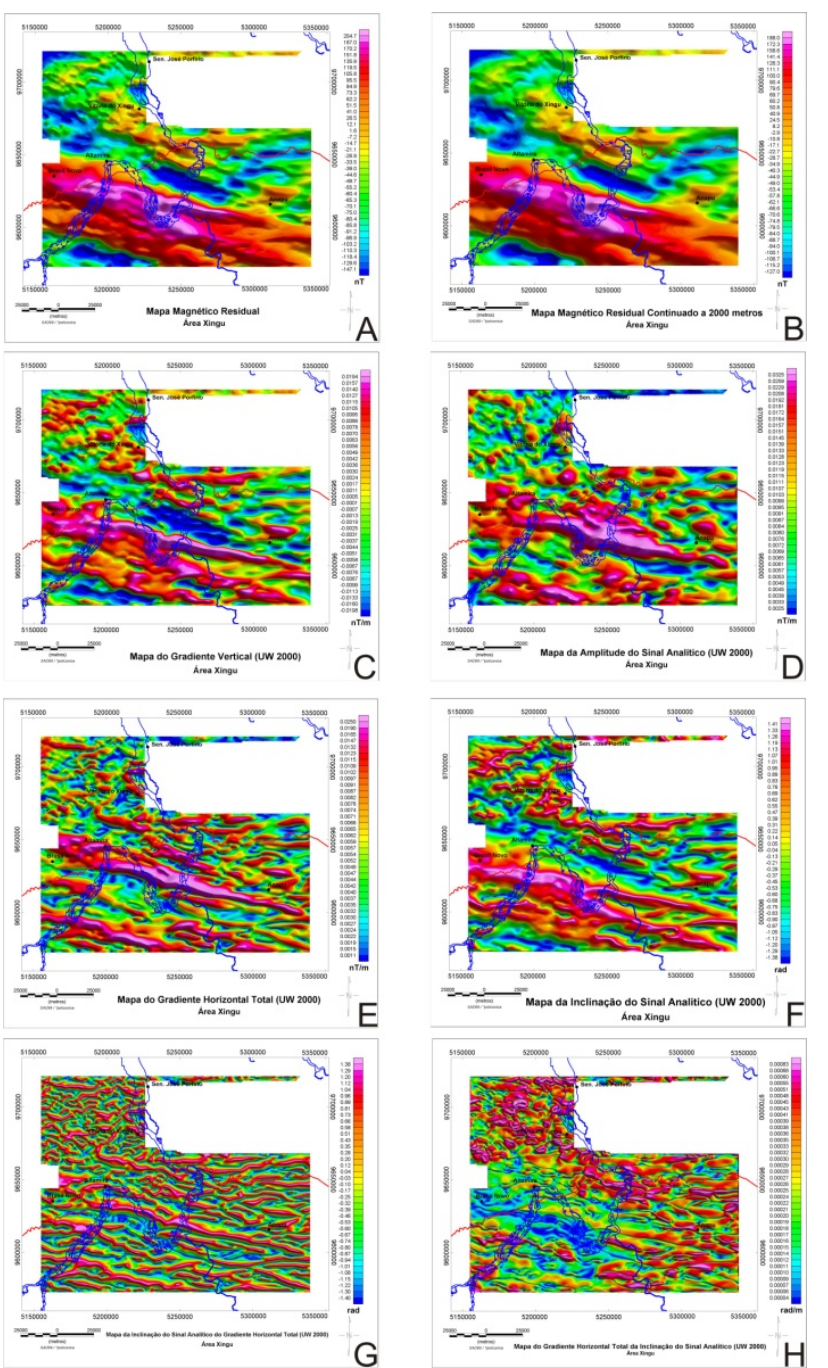

B

(1)

\section{政}




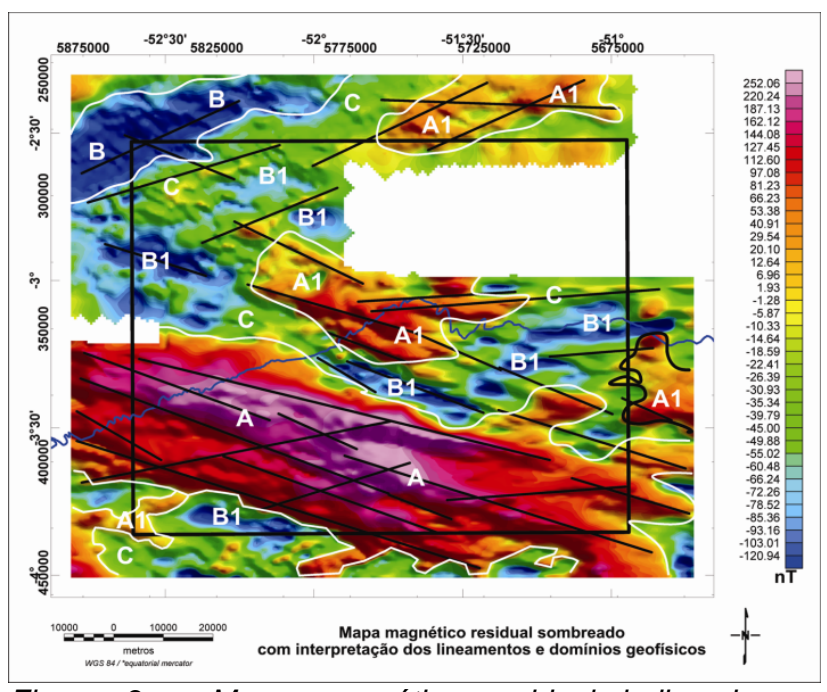

Figura 3 - Mapa magnético residual indicando os principais domínios, subdomínios e lineamentos geofísicos (em preto a área de estudo e lineamentos, em azul o contato embasamento/bacia e em branco os domínios).

Os mapas do gradiente vertical (Gz, Fig. 2-C) e da inclinação do sinal analítico (ISA, Fig. 2-F) foram utilizados para a interpretação dos eixos dos corpos, sendo possível observar o refinamento da estruturação predominantemente WNW e NW, contínua no domínio do embasamento, e a presença de estruturas mais segmentadas de direção $\mathrm{NE}$, no contexto da bacia. O mapa do gradiente horizontal total (GHT, Fig. 2-E) foi utilizado na tentativa de delimitar os limites dos corpos magnéticos.

O mapa da amplitude do sinal analítico (ASA, Fig.2-D) permitiu localizar alguns corpos, mostrando um trend NW próximo ao contato com a bacia, porém mais proeminente no embasamento. A dificuldade de visualização das estruturas na bacia se deve, provavelmente, a maiores profundidades das fontes, o que implica em atenuação das amplitudes do sinal, como demonstrado através de modelos sintéticos de ASA (Ferreira et al., 2010, neste simpósio).

O mapa do gradiente horizontal total da inclinação do sinal analítico (GHT-ISA, Fig. 2-H), apesar de realçar algumas estruturas no contexto da bacia e ser independente da inclinação magnética (Verduzco et al. 2004), não exibe uma resposta satisfatória para a interpretação magnética. Já o mapa da inclinação do sinal analítico do gradiente horizontal total (ISA-GHT, Fig.2-G), Ferreira et al. (2010), neste simpósio, revelou boa correlação as estruturas do embasamento de direções NW e WNW, além de igualmente realçar as tendências no interior da bacia, fato não claramente evidenciado pelos demais métodos.

A interpretação das principais tendências estruturaismagnéticas é apresentada na Figura 4.

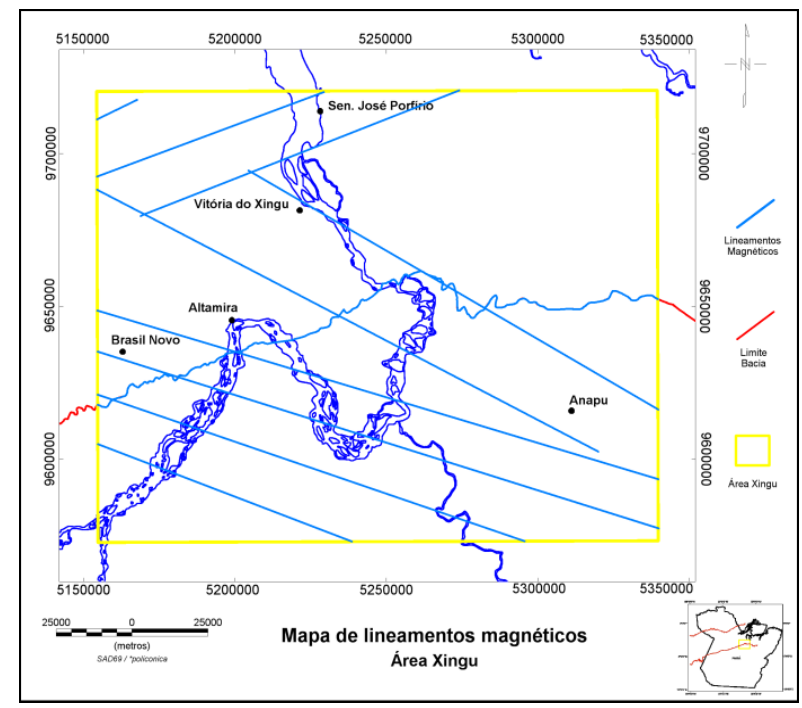

Figura 4 - Mapa de lineamentos magnéticos da área.

A partir do mapa da Figura 4 foram elaborados diagramas de roseta de frequência e comprimento dos lineamentos geofísicos. As direções preferenciais N60$80 \mathrm{~W}$ para comprimento dos lineamentos e N6080W/N60-70E para freqüência são indicadas na Figura 5. Estas direções revelam grande correspondência aos dados estruturais mapeados por diversos autores (CPRM 2008).

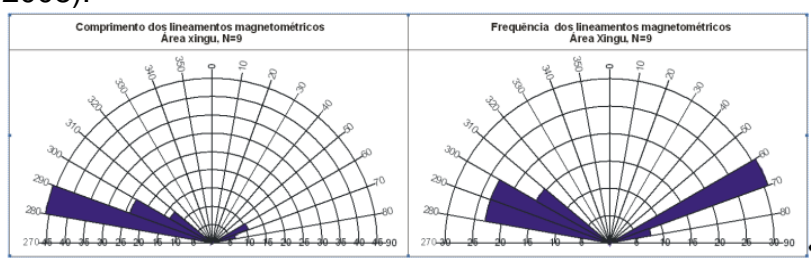

Figura 5 - Diagramas de roseta de comprimento e frequência acumulados dos lineamentos geofísicos da área de estudo.

De acordo com o espectro de potência radial (Fig. 6), as fontes profundas apresentam comprimento de onda maior que $12,5 \mathrm{~km}$ e profundidades superiores a $7,7 \mathrm{~km}$, correspondentes a estruturas do embasamento. As fontes intermediárias são discriminadas entre os comprimentos de onda de $12,5 \mathrm{~km}$ e $3,5 \mathrm{~km}$, representando profundidades entre 7,7 e 4,3 km, também relativas a estruturas do embasamento. Já as fontes rasas exibem comprimentos de onda entre 3,5 a 2,2 km e profundidades variáveis de 1,7 a 4,3 km, estas podendo se relacionar a estruturas da Bacia do Amazonas.

O método da deconvolução de Euler foi aplicado aos dados magnéticos residuais da Figura 3, para o modelo dique, como mostra a Figura 7, na qual foram sobrepostos os lineamentos magnéticos obtidos a partir da interpretação qualitativa (Fig. 4).

De acordo com a Figura 7, as profundidades variam desde menores que $1,4 \mathrm{~km}$ a superiores a $5,0 \mathrm{~km}$. As soluções são paralelas ao trend NW/WNW delineado na Figura 4, com profundidades compatíveis ao embasamento raso adentrando a bacia. 


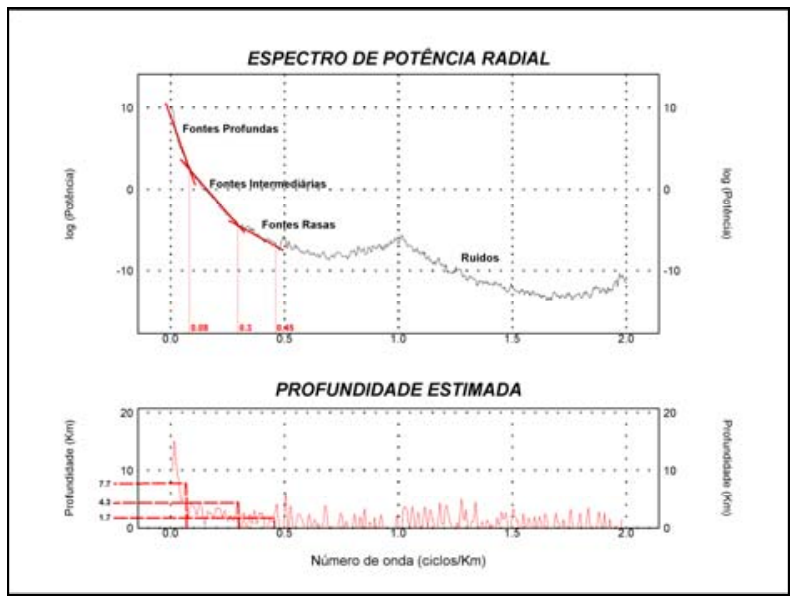

Figura 6 - Espectro de potência radial gerado a partir do mapa magnético residual.

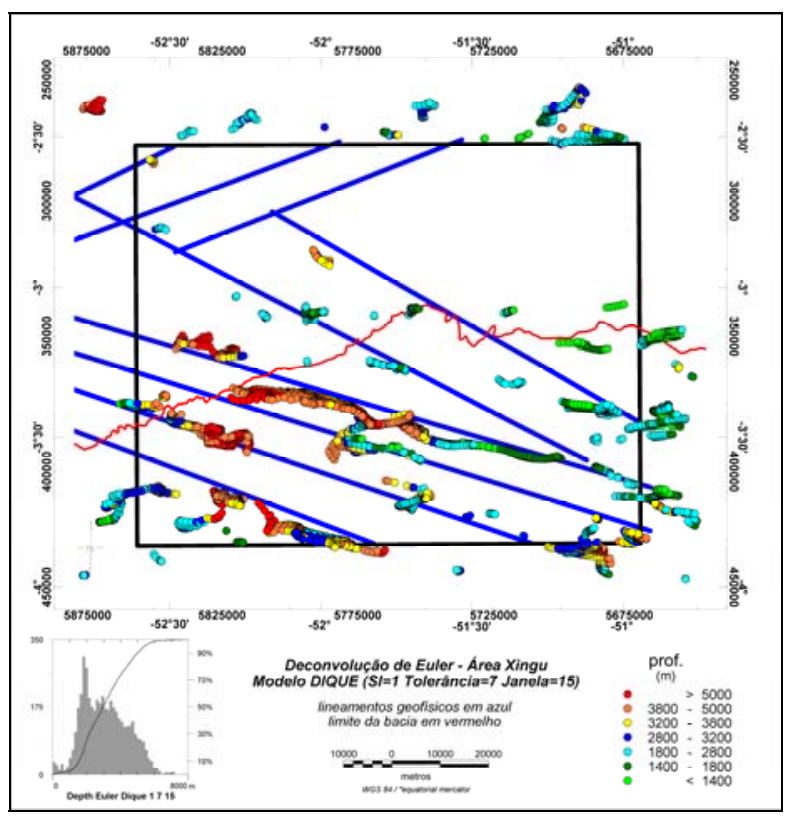

Figura 7 - Mapa das soluções de Euler para o modelo dique.

\section{Discussão e Conclusões}

O trend WNW de grandes amplitudes do mapa magnético residual (Domínio A, Fig. 3), quando comparado ao mapa geológico da CPRM (2009), reflete rochas do embasamento (Granodiorito Oca, Micaxisto Bacajá, Granitóide Bacajaí e Anfibolito Itatá).

Observou-se, de acordo com as figuras 4 e 5 , que os lineamentos geofísicos têm direções preferenciais NW, subordinadamente NE, contínuas no embasamento e interrompidas na Bacia do Amazonas. A área apresenta um forte domínio das estruturas NW e WNW do embasamento que continuam na bacia.

O mapa continuado para 2000 metros (Fig. 2-B) respondeu de forma satisfatória à etapa de filtragem, eliminando em grande parte os ruídos e preservando os sinais. Os mapas do gradiente horizontal total (Fig. 2-E) e inclinação do sinal analítico (Fig.2-F) mostram uma boa correlação com as estruturas de superfície,.assim como o mapa da amplitude do sinal analítico (Fig.2-D). O mapa de inclinação do sinal analítico do gradiente horizontal total (Fig.2-G) revelou grande aplicabilidade na identificação de estruturas.

A análise espectral mostrou que as estruturas profundas e intermediárias com profundidades de 7,7 a 4,4km são relacionadas a estruturas do embasamento profundo a raso. Da mesma forma, a deconvolução de Euler exibiu soluções que corroboram os lineamentos geofísicos, cujas profundidades são compatíveis ao embasamento exposto e oculto sob a Bacia do Amazonas.

\section{Agradecimentos}

Os autores agradecem a Petrobras pelo financiamento da pesquisa e a CAPES pela cessão de bolsa de doutoramento concedida a A.B.S. Bongiolo. F.J.F. Ferreira agradece ao CNPq pela bolsa de Produtividade em Pesquisa.

\section{Referências}

CORDELL L \& GRAUCH VJS. 1985. Mapping basement magnetization zones from aeromagnetic data in the San Juan Basin, New México. In: Hinze WJ (ed). The Utility of Regional Gravity and Magnetic Anomalies Maps. Society of Exploration Geophysics, p. 181-197.

CPRM 2008. Geologia e Recursos Minerais do Estado do Pará. Escala 1:1.000.000. Belém, 328p.

CPRM 2009. Mapa Geológico do Brasil, Escala 1:1.000.000. Companhia de Pesquisas e Recursos Minerais.

CUNHA PRC, GONZAGA FG, COUTINHO LFC \& FEIJÓ FJ. 1994. Bacia do Amazonas. Boletim de Geociências Petrobras, 8(1):47-67.

EVJEN HM. 1936. The place of vertical gradient in gravitacional interpretations. Geophysics, 1:127-136.

FERREIRA FJF, SOUZA J de, BONGIOLO ABS, CASTRO LG de \& ROMEIRO MAT. 2010. Realce de anomalias magnéticas do gradiente horizontal total usando a inclinação do sinal analítico. Parte I - Aplicação a dados sintéticos (neste simpósio).

JOÃO XSJ, VALE AG \& LOBATO TAM. 1987. Projeto Altamira - Folha SA.22-Y-D. Programa de Levantamentos Geológicos Básicos, Serviço Geológico do Brasil (CPRM), 31 p. Brasília.

LI X. 2008. Magnetic reduction-to-the-pole at low latitudes: Observations and considerations. The Leading Edge, 27(8): 990-1002.

MILLER HG \& SINGH V. 1994. Potential field tilt - a new concept for location of potential field sources. Journal of Applied Geophysics, 32:213-217. 
NABIGHIAN MN. 1972. The analytic signal of twodimensional magnetic bodies with poligonal cross-section: its properties and use for automated anomaly interpretation. Geophysics, 37:507-517.

PERICO E. 2009. Análise estrutural multiescala da província Maroni-Itacaiúnas e da Bacia do Amazonas na região de Altamira, Estado do Pará. Dissertação (Mestrado), Pós-Graduação em Geologia, Universidade Federal do Paraná, Curitiba (em preparação).

ROEST WRJ, VERHOEF J \& PILKINGTON M. 1992. Magnetic interpretation using the 3-D analytic signal, Geophysics, 57:116-125.

SPECTOR A \& GRANT FS. 1970. Statistical models for interpreting aeromagnetic data. Geophysics, 35:293-302.

TASSINARI CGC \& MACAMBIRA MJB. 1999. Geochronological provinces of the Amazonian Craton. Episodes, 22(3):174-182.

TASSINARI CCG \& MACAMBIRA MJB. 2004. A evolução tectônica do Cráton Amazônico. In: V. Mantesso Neto, A. Bartorelli, C.D.R. Carneiro, B.B. Brito-Neves (eds.) Geologia do Continente Sul-Americano: Evolução da obra de Fernando Flávio Marques de Almeida. São Paulo, Ed.Beca, p.471-485.

THOMPSON DT. 1982. EULDPH: A new technique for making computer assisted depth estimates from magnetic data. Geophysics, 47(1):31-37.

VERDUZCO B, FAIRHEAD C, GREEN, C \& MACKENZIE C. 2004. New insights into magnetic derivatives for structural mapping. The Leading Edge, 23:116-119. 
IV Simpósio Brasileiro da SBGf - Brasília 2010 